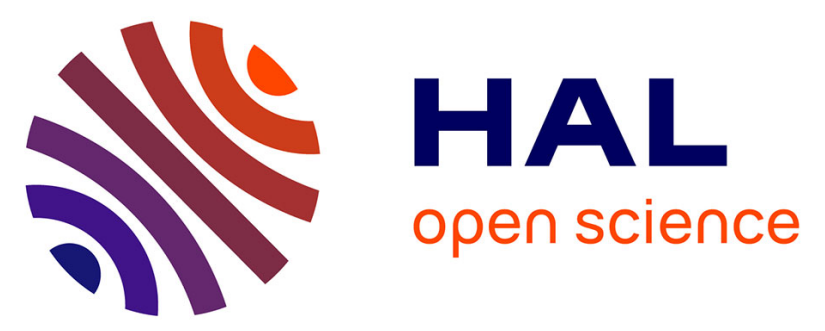

\title{
Relations entre la perméabilité en fréquence et les propriétés structurales des alliages Co-Fe-Si-B déposés par pulvérisation ionique
}

J. Vermeulen, O. Acher, F. Ravel

\section{- To cite this version:}

J. Vermeulen, O. Acher, F. Ravel. Relations entre la perméabilité en fréquence et les propriétés structurales des alliages Co-Fe-Si-B déposés par pulvérisation ionique. Journal de Physique IV Proceedings, 1992, 02 (C3), pp.C3-235-C3-238. 10.1051/jp4:1992335 . jpa-00251540

HAL Id: jpa-00251540

https://hal.science/jpa-00251540

Submitted on 1 Jan 1992

HAL is a multi-disciplinary open access archive for the deposit and dissemination of scientific research documents, whether they are published or not. The documents may come from teaching and research institutions in France or abroad, or from public or private research centers.
L'archive ouverte pluridisciplinaire HAL, est destinée au dépôt et à la diffusion de documents scientifiques de niveau recherche, publiés ou non, émanant des établissements d'enseignement et de recherche français ou étrangers, des laboratoires publics ou privés. 


\title{
Relations entre la perméabilité en fréquence et les propriétés structurales des alliages $\mathrm{Co}-\mathrm{Fe}$-Si-B déposés par pulvérisation ionique
}

\author{
J.L. VERMEULEN, O. ACHER et F. RAVEL
}

Commissariat a l'Energie Atomique, Centre d'Etudes de Bruyères le Chatel, BP. 12, 91680 Bruyeres le Chatel, France

\section{Abstract:}

In the present study, structural properties and microwave permeability of $\left(\mathrm{Co}_{88} \mathrm{Fe}_{9} \mathrm{Si}_{3}\right)_{100-x} B_{x}(5 \leq x \leq 15$ at.\%) films prepared by ion beam sputtering (IBS) were investigated as a function of film composition and deposition parameters. $A$ microcrystalline phase with $\langle 111\rangle$ orientation normal to the film plane is obtained for low boron content $(x<6 a t . \%)$ and deposition rate above $2 \mathrm{~nm} / \mathrm{min}$, whereas the material is amorphous or partially crystallized at $1,5 \mathrm{~nm} / \mathrm{min}$ or for a boron content of 12 at.\%. In-plane uniaxial anisotropy field $H_{k}$ and coercive force $H_{c}$ have also been found to be strongly dependent on deposition rate. For microcrystalline films prepared at high growth rate, permeability measurements in the gigahertz range show that gyromagnetic resonance frequency is $1.5 \mathrm{GHz}$ whereas the real part of relative permeability $\mu^{\prime}$ is 1100 along the hard axis and 1 along the easy axis. In contrast amorphous films deposited at low deposition rate exhibit semihard properties. The complex permeability spectra shows a low rotational permeatility $\mu^{\prime}(<200)$ combined with a high resonance frequency more than $3 \mathrm{Ghz}$. This behavior might be attributed to the structural inhomogeneities in alloy.

\section{I - Introduction}

Les alliages $\mathrm{CoFeSiB}$ à faible teneur en métalloïdes (<10\%at.) suscitent actuellement un intérêt grandissant dans le domaine de l'enregistrement magnétique et des applications hautes fréquences. Cet engouement est en partie lié à leur structure microcristalline qui leur confère des propriétés magnétiques douces [1-3]: faible coercitivité $\left(H_{C}=0.2 \mathrm{Oe}\right)$, induction à saturation supérieure à celle des amorphes magnétiques $\left(B_{S}>16000\right.$ Gauss) et forte perméabilité dynamique $\left(\mu^{\prime} \approx 500-1100 \mathrm{a} 500 \mathrm{MHz}\right)$ dont la valeur dépend des conditions d'élaboration des échantillons, de la concentration en métallö̈de mais surtout du champ anisotropie $H_{k}$ qui leur physiquement corrélé.

Au travers de cet article, nous examinons le rôle particulier de deux paramètres importants: la concentration en bore et la vitesse de dépôt $v$. Nous tentons de les relier aux évolutions de la microstructure et à celles de la perméabilité magnétique en fréquence.

\section{II - Procédure d'élaboration et caractérisations}

Les couches sont réalisées dans un bâti de pulvérisation ionique. Elles sont obtenues par pulvérisation directe de l'alliage en utilisant un flux de particules $\mathrm{Ar}^{+} \mathrm{d}^{\prime} 1 \mathrm{keV}$. La configuration générale de l'installation est décrite ailleurs $[3,4]$. Plusieurs cibles de concentrations différentes ont été utilisées pour cette étude.

L'opération s'effectue sous vide secondaire $\left(3.10^{-5}\right.$ Torr), la pression résiduelle n'excèdant pas $10^{-7}$ Torr. Au cours du dépôt, les substrats subissent un échauffement par l'émission électronique du neutraliseur. Leur température est évaluée à l'aide d'un thermocouple placé sur un substrat de référence: elle varie entre 50 et $120^{\circ} \mathrm{C}$. Afin d'obtenir une meilleure uniformité d'épaisseur et de composition, les dépôts sont réalisés sur substrats de silice fondue en rotation (1.5 tour $/ \mathrm{sec})$. Leur vitesse de croissance varie entre 1,2 et $2,5 \mathrm{~nm} / \mathrm{min}$ et les films 
sont affectés d'une contrainte de compression. L'amplitude de cette contrainte, déduite du changement de courbure des substrats, est comprise entre $-1.10^{9}$ et $-10.10^{9}$ dynes $/ \mathrm{cm}^{2}$.

Les mesures de composition des couches déposées sont réalisées par microanalyse à la microsonde de Castaing. Les grandeurs magnétiques statiques $H_{k}$ et $H_{c}$ ainsi que la visualisation des domaines magnétiques sont obtenues par Effet Kerr magnéto-optique et par microscopie de Lorentz [5]. Les perméabilités dynamiques $\mu^{\prime}$ et $\mu^{\prime \prime}$ sont mesurées par la méthode de perturbations de spires [6]. Enfin, des caractérisations en diffractométrie $X$, au goniomètre à texture ainsi qu'au microscope électronique en transmisssion complètent l'étude.

\section{III - Résultats et discussions}

\section{1 - Propriétes structurales}

\section{* Effets des additions croissantes de bore dans l'échantillon}

L'analyse radiocristallographique effectuée sur un alliage $\left(\mathrm{Co}_{88} \mathrm{Fe}_{9} \mathrm{Si}_{3}\right)_{100-x} \mathrm{~B}_{x}$ déposé à $v=1.7 \mathrm{~nm} / \mathrm{min}$ montre que le taux de cristallisation des couches est fonction décroissante de la quantité de bore présent dans l'alliage. De 5 à $6 \%$ at, le matériau est microcristallisé et présente une texture (111) cfc perpendiculaire au plan du film. La taille des cristallites déterminée à partir des spectres de diffractométrie X par la méthode de Scherrer est comprise entre 10 et $20 \mathrm{~nm}$ et la désorientation des axes $\langle 111\rangle$ est inférieure à $6^{\circ}$. Pour des taux de substitution plus importants, (i.e entre de $6 \%$ à $12 \%$ at.), l'alliage perd progressivement sa structure cristalline. $\mathrm{Sa}$ taille de grain diminue et passe en dessous de $8 \mathrm{~nm}$; simultanément, la désorientation des cristallites s'accentue et atteint $12^{\circ}$. Au delà de $12 \%$ at., plus aucun pic de diffraction n'apparait sur les spectres de rayon $X$, indiquant que le matériau est devenu pratiquement amorphe.

* Influence de la vitesse de dépôt.

L'augmentation de la vitesse de dépôt dans la gamme $1,2<v<2,5 \mathrm{~nm} / \mathrm{min}$ a pour effet d'accroître la concentration seuil d'amorphisation en bore, passant ainsi de $12 \%$ at.pour une vitesse de $1,2 \mathrm{~nm} / \mathrm{min}$ à $15 \%$ at. pour $2,5 \mathrm{~nm} / \mathrm{min}$. Elle entraine également une élévation de la température du substrat de $50^{\circ} \mathrm{C}$ à $90^{\circ} \mathrm{C}$.

\section{2 - Propriétés magnétiques}

L'ensemble des carctérisations pratiquées sur nos échantillons montre que, dans la gamme de composition étudiée $(5<x<12 \%$ at $)$ les propriétés des couches sont peu influencées par la concentration de bore. En revanche, celles-ci se sont révélées étroitement liées à la vitesse de croissance.

A $v=2,5 \mathrm{~nm} / \mathrm{min}$ et pour $x=6.9 \%$ at., les couches sont magnétiquement douces et sont caractérisées par un axe de facile et de difficile aimantation dans leur propre plan. La coercitivité mesurée à l'hystérésimètre Kerr est faible et toujours inférieure à 0.5 Oe. Par ailleurs, les observations de domaines au microscope à effet Kerr montrent que l'anisotropie uniaxiale est relativement homogène à l'échelle de l'échantillon, aussi bien en intensité $\left(\Delta H_{k} / H_{k} \approx 4 \%\right)$ qu'en direction $\left(\Delta \phi \leq 10^{\circ}\right.$ autour de la direction facile). En outre, son intensité moyenne est fonction décroissante de la vitesse de croissance et varie entre 13,5 Oe et 33 Oe.

La perméabilité complexe en fréquence $\mu^{*}$ est gyromagnétique à caractère résonant $(\alpha \sim 500-850 \mathrm{MHz})$ avec une fréquence de résonance située entre 1,5 et $3 \mathrm{GHz}$ selon l'intensité du champ d'anisotropie $H_{k}$. La perméabilité réelle selon l'axe "difficile" est relativement élevée $\left(\mu^{\prime} \sim 500-1100\right)$ et ce jusqu'à des fréquences de l'ordre du gigahertz (fig 1). Elle vaut en revanche l'unité selon l'axe de facile aimantation.

A l'opposé, pour les faibles vitesses de croissance $(v=1,2 \mathrm{~nm} / \mathrm{min})$ et toujours pour les mêmes teneurs en bore, les films élaborés sont amorphes et présentent des propriétes dynamiques plutôt médiocres. L'hystérésigraphie Kerr appliqués sur sur ces échantillons révèlent que l'anisotropie dans le plan de la couche a pratiquement disparu (fig 2). Cette analyse 


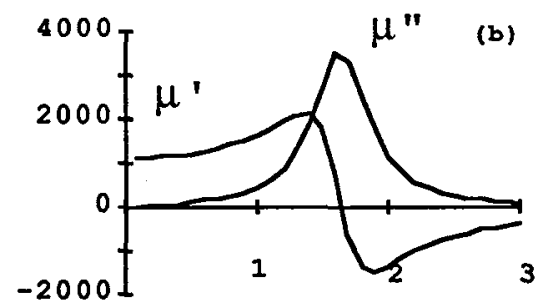

figure 1: spectres de perméabilité complexe selon l'axe de difficile aimantation d'un échantillon élaboré à 2,5 $\mathrm{nm} / \mathrm{min} . \mu^{*}=0$ selon l'autre direction

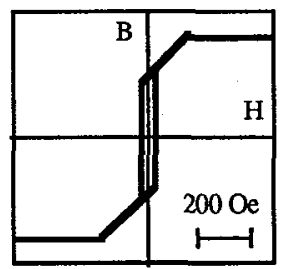

figure 2: cycle d'hystérésis obtenu par effet Kerr sur une couche élaboré à faible vitesse de dépôt (v=1.2 nm).

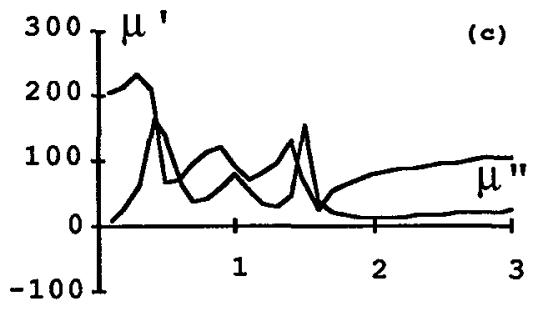

figure 3: spectre de perméabilité complexe obtenu par la méthode de perturbation de spires sur un film préparé a $v=1,2 \mathrm{~nm}$. Les niveaux de perméabilité mesurés sont pratiquement identiques quelle que la direction de mesure dans le plan de la couche

est confirmée par la mesure des spectres de perméabilité en fréquence, indiquant que les propriétés des films sont devenues quasi-isotropes dans leur plan (fig 3).

Un tel comportement, déjà observé sur des couches amorphes de CoZr et de CoNbZr [7], ne peut êtte attribué qu'à l'existence d'un certain champ d'anisotropie perpendiculaire au plan du dépôt, ou encore à la présence de fortes inhomogénéités chimiques dans l'alliage. Pour s'en persuader, une serie d'observations au microscopie électronique en transmission ont donc été réalisées sur nos échantillons. Les clichés obtenus sont reportés sur la figure 4. On peut voir que la direction de l'aimantation est très fluctuante à l'échelle du micron. De même, l'homogénéité chimique et structurale de l'alliage à l'état brut de dépôt ne semble pas parfaite. Ces déficiences sont probablement dues aux faibles vitesses de croissance qui, en limitant la mobilité des atomes de surface, favorisent la formation d'une morphologie colonnaire. Cette hypothèse n'a malheureusement pas pu être vérifiée au microscope électronique à balayage (en raison de la faible épaisseur des couches). Néanmoins les mesures de densité effectuées sur l'ensemble de nos échantillons indiquent qu'une légère porosité apparait à faible vitesse de dépôt. 
(a)

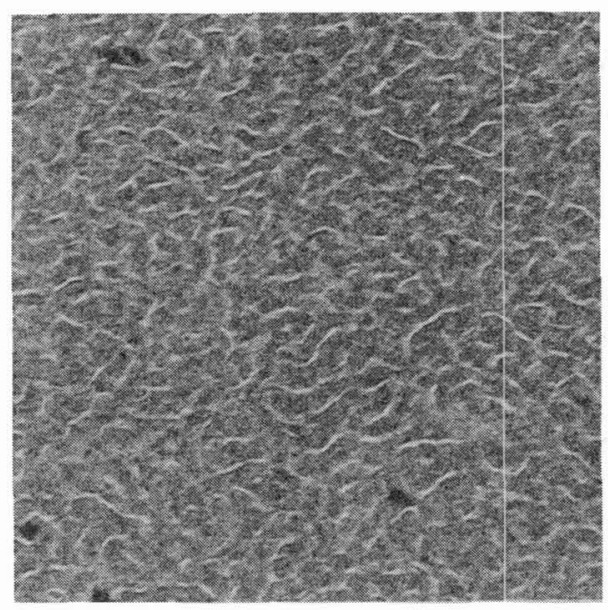

$20 \mathrm{~nm}$ (b)

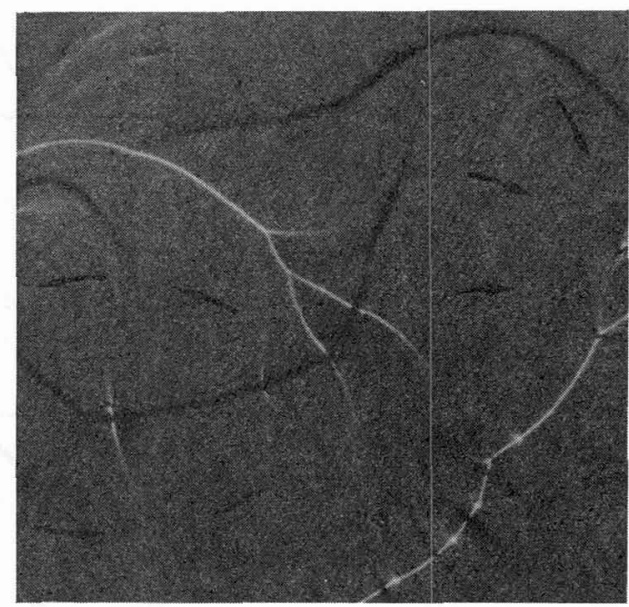

$2 \mu \mathrm{m}$

figure 4: (a) Micrographie électronique représentant la surface d'un échantillon élaboré à v=1,2 nm; (b) visualisation en microscopie de Lorentz des domaines magnétiques associés .

\section{IV - Conclusions}

Au travers de cette étude, nous nous sommes attaché à mettre en évidence l'influence de la concentration de bore et des conditions d'élaboration sur les propriétés structurales et magnétiques des couches de $\left(\mathrm{Co}_{88} \mathrm{Fe}_{9} \mathrm{Si}_{3}\right)_{100 \cdot x} \mathrm{~B}_{x}$ brutes de dépôt. Il est apparu que la perméabilité en fréquence de l'alliage était très sensible à la microstructure et, dans une certaine mesure, de la vitesse de dépôt. Des couches microcristallisées ont été obtenues aux vitesses de croissance les plus élévées. Elles présentent les meilleures propriétés en fréquence avec des perméabilités réelles $\mu^{\prime}$ atteignant 1100 et ce, jusqu'à des fréquences supérieures au gigahertz. Nous avons constaté en revanche que la diminution de la vitesse de dépôt dégradait de manière significative les propriétés en fréquence des couches. Nous avons attribué ce phénomène à l'existence d'hétérogénéités structurales au sein de l'alliage responsable de la formation d'une anisotropie perpendiculaire au plan du dépôt.

\section{Remerciements}

Les auteurs tiennent à remercier C. Boscher pour sa participation active aux expérimentations ainsi que L.Mancheron pour la relecture critique de ce manuscrit.

\section{Références}

[1] - F.W.A Dirne and M. Brouha, I.E.E.E Trans. on Mag., 24, 2, (1988) 1862.

[2] - F.W.A Dirne and M. Brouha, Mat. Sc. Eng., 99, (1988) 57.

[3] - J.L. Vermeulen, C. Boscher, rapport interne.

[4] - J.L. Vermeulen, O. Acher, C. Barbalat, Le Vide Les Couches Minces, 3rd Symposium on Trends and New Applications in Thin Films, $n$ 259, 164 (1991).

[5] - Harrison W. Fuller and Murray E. Hale, J. App. Phys., 31 (1960) 238.

[6] - J.C. Peuzin, J.G. Gay, Acte des journées d'études sur la caractérisation microonde des matériaux absorbants, Limoges (1991) 75

[7] - A. Materne, These de Physique, Grenoble (1987). 